\title{
ZUM DEUTSCHEN VERBUM.
}

1. Die endung der ersten person pluralis.

Von literatur über den gegenstand nenne ich Scherer z. gesch. s. $189 \mathrm{ff}$. (=2 $299 \mathrm{ff}$.), A. Kuln in seiner zs. 18, $332 \mathrm{ff}$, Paul beitr. 4, 404. 421 ff. Besonders wichtig sind die materialsammlungen in Graffs sprachschatz 2, $574 \mathrm{ff}$. $966 \mathrm{f}$, , die, soweit sie aus noch nicht veröffentlichten glossarien stammen, auch der folgenden darstellung zu grunde liegen. Meine eigenen annahmen in der schrift über das Keronische glossar s. $182 \mathrm{f}$. scheinen mir nicht alle mehr haltbar, während auf die dort s. $181 \mathrm{ff}$. zusammengestellten formen öfter zu verweisen sein wird. Vom vergleichenden standpunkte aus ist das suffix der ersten pers. plur. act. behandelt von Brugman morph. unters. I, $151 \mathrm{ff}$.

A) Ueber das verhältnis von ahd. $-m e \hat{s}$ zu dem $-m$ des gotischen und nordischen (die nördlichen zweige des westgermanischen, sächsisch, friesisch, englisch besitzen die alte form der 1. pl. bekanntlich nicht mehr) hat zuletzt Paul a. a. o. gehandelt. Er kommt auf die meinung Kuhns zurïck, dass das urgermanische nur diejenige form besessen habe, welche im ostgermanischen vorliegt, $-m$ aus ${ }^{*}-m e n$, während die in alı. erscheinende endung ein suffigiertes pronomen der ersten person, das dem got. veis entsprïche, in sich enthalte. Er glaubt Kuhns beweise noch um einen vermehren zu können, da er beobachtet hat, dass im $\mathrm{T}$. hinter der form auf -mês das pronomen uuir gewöhnlich fehlt: 'T. sagt nemlich nicht giloubemes uuir, wol aber uuir giloubemes und gilouben uuir. Indes wird durch diese tatsache meiner ansicht nach nichts weiter bewiesen, als dass die sprechenden in dem -mês noch die kraft 
eines uuir fühlten. Diese muss aber doch auch dann in dem suffixe gelegen haben, wenn es aus der ursprache ererbt ist; denn die hinzusetzung der personalpronomina zu den verbalformen ist ja doch nur cine folge der lautlichen abschwächung der endungen, ein ersatz für die verlorenen bestandteile, denen die bedeutung der personalpronominen innegewohnt hatte. Wo aber das suffix deutlich genug geblieben war, brauchte man es eben nicht durch hinzufügung des personalpronomens aufzufrischen. Wir haben es also hier nur mit einer erhaltenen syntaktischen altertümlichkeit $\mathrm{zu}$ tụn, die für die erklärung des suffixes gleichgültig ist. Paul ibersieht ja auch keineswegs, dass der Kuhnschen auffassung unüberwindliche schwierigkeiten im wege liegen, da das $\hat{e}$ statt $\hat{\imath}$ und die erhaltung des $s$ den bekannten lautgesetzen zuwiderlaufen, auch wäre wol $m m$ zu erwarten.

Mit recht hat daher Joh. Schmidt in seinem vocalismus II, 279 die Kuhnsche erklärung verworfen und ist auf die alte schon von Bopp gegebene zurlickgekommen, wonach -mês mit dern vedischen -masi identisch ist. Schmidt führt -mês ohne zweifel richtig auf -mais zurück, weil alle übrigen $\hat{e}$ in althochd. endsilben (sobald die länge bis in die historische zeit hineinreicht) auf früheres ai hinweisen; da aber die erhaltung des $s$ zwingt, den verlust eines auslautenden vocals anzunehmen, so kann die urgermanische gestalt des suffixes nur -maisi gewesen sein, und von da bis $\mathrm{zu}$ ved. -masi ist nur noch ein schritt. Die epenthese macht keine schwierigkeit, wenn auch die genaueren bedingungen, unter welchen sie eintritt, noch nicht ermittelt sind: denn einen ganz analogen fall erkennt man leicht in ahd. n. sg. m. blintêr aus *blindazì, vgl. gr. oi $\tau o \sigma \hat{\imath}$, $\dot{\varepsilon} x \varepsilon$ voo $\dot{\imath}$, skr. $\hat{\imath}$, zd. hyati (Fick 1, 505), wo also das $\bar{\imath}$ demonstrativum, das ahd. im auslaut schwinden musste, wie das $-\bar{\imath}$ im n. sg. der jâ-stämme (vgl. Ker. gl. s. 26 indechnes apocalypsis, thicnes densitas, aus *-nessí) an den n. soc. m. des adjectirs (ursprünglich wol gewisser pronomina, wie jenêr) angetreten ist, natürlich lange vor der wirkung des conson. auslautş̧esetzes. Für diese annalme ist natürlich kein hinderniss, dass die form auf - $\hat{e} r$ nur in einem eiuzigen dialektc vorkommt, denn die aufugung des verstärkenden $-\overline{\grave{\imath}}$ war von allem anfang an facultativ ebenso wie im griechischen, und die einfache 
form blint aus *blindas besteht ja auch im ahd. noch daneben. Was Sievers beitr. II, 103 uber die form blintèr bemerkt, wird er jetzt nicht melir aufrecht erhalten wollen, nachdem die sprachhistorischen erfahrungen, die seitdem bedeutend erweitert sind, uns belebrt haben, dass sehr wol eine einzelne mundart eine altertumlichkeit bis in sehr späte zeit hinein bewahrt haben kann. Auch das dritte der bisher unerklärten $\hat{e}$ in althd. endsilben, im g. pl. des pron. pers. unsêr iuuêr (vgl. Braune beitr. 2, $140 \mathrm{ff}$.) muss auf ai zurückgehen und da durch got. unsara izvara $r$ sich als urgermanisch erweist, so wird wol die germ. grundform kaum anders als unsarı́ inarí (auf die gestalt der wurzelsilbe kommt es nicht an) anzusetzen sein.

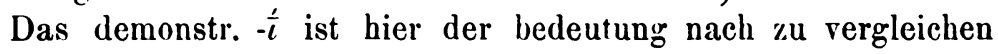
mit dem got. anfitgsel $a$ und dem $-h a$ in ahd. ihha (vgl. gr.

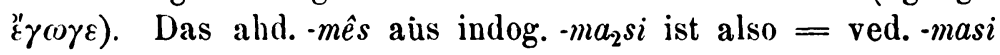
altb. -mahi altp. -mahy, und es ist hier die alte indogermanische primärform des suffixes der 1. pl. act. (oder eine der primärformen, denn gr. $-\mu \varepsilon \varsigma$, lat. $-m u s=$ serb. $-m o$ ir. $-m$ lassen sich nicht mit -masi vereinigen) bewahrt geblieben. Die secundärform dazu ist $-m$ aus *-men, vgl. gr. $-\mu \varepsilon v$ skr. $-m a$ lit. $-m e$. Im ostgermanischen ist wie im attischen und litauischen (wol auch altbulgarischen) die secundärform verallgemeinert, im ältesten ahd. aber sind primäre und secundäre form in ihren functionen noch geschieden, während später auch hier uberall die kürzere, bequemere secundärform durchdringt; in einzelnen mundarten scheint cine zeitlang die primärform den sieg elringen zu wollen, wird aber auch da schliesslich zurickgedrängt.

Die ältesten althd. denkmäler haben -mês nur im ind. praes., nicht aber im opt. praes. und im praeter. iud. und opt., wo sie vielmehr -m setzen. Die hymnen geben dieses verhältnis am reinsten wider. Hier hat der ind. praes. stets -mês (mehr als $30 \mathrm{mal}$ ), der opt. praes. auch in adhortativer anwendung ebenso regelmässig stets $-m$ (42 mal). Präteritalformen sind leider nicht belegt, denn auf 26, 15, 2 ist nicht viel zu bauen. In den drei handschriften des Keronischen glossars sind im ind. praes. nur formen auf -mês belegt (abgesehen von pirum, woruber unten), dagegen hat der ind. praet. nur -m (üb. d. Ker. gl. s. 189); optative des prät. 
fiehlen leider, aber im optativ des präs. steht vielleicht schon $-m \hat{s}$, wemn hier nicht etwa ungenaue übersetzungen vorliegen. In der benedictinerregel kommt der opt. praet. nicht vor, der opt. praes. aber endet stets auf $-m$ (qhuememees 35,2 veniamus ist adhortativus, der in diesem denkmal durchweg auf -mês ausgeht, vgl. Seiler beitr. I, 452), während der indic. präs. nur - mềs aufiveist, das sich aber hier bereits auf den ind. prät. verbreitet hat. Im Isidor und in den fragm. theot. hat das prät. ind. und opt. nur -m, das präs. nur -mês, aber auch schon im optativ. In den fragm. haben auch die prät.-präs. -mês. Das glossar R. endlich folgt in den belegten formen durchweg der regel: praes. ind. -mês (adhort. -mês: zaprechumes 113,15), opt. fehlt, praet. ind. und opt. $-m$. Ebenso die gl. Cass. In den ubrigen denkmälern herscht entweder ausschliesslich -mês (so in Rd-Ib., Rb., Ja., Jc.), oder wenn $-m(-n)$ daneben vorkommt, ist die alte regel verwischt (so bei 0 . und T.). Ganz selten kommt in älteren quellen $-m$ im ind. praes. vor; Graft a. a. 0. bringt nur 4 derartige beispiele bei, wovon drei auf den Weissenburger catechismus entfallen, der liberhaupt vielmehr - $m$ als -mês hat und so den späteren stand vorbereitet, und eins auf das S. Galler pater noster (oblazem), welches wol auf rechnung der (fränkischen?) vorlage kommt. Also:

Opt. praes. nur $-m$ : Hymn. B.-R. ${ }^{1}$ )

Ind. praet. $n \quad n$ Pa. gl. K. Ra. R. Cass. Is. frg.

Opt. praet. $n \pi$ R. Cass. Is. Frg.

Ueberall im gegensatz zum ind. praes. und teilweise zum adhortativus (der dann mit dem ind. gleichlautet), wo sich nur -mês findet.

Eine ausnahme macht pirum, das in allen älteren deukmälern auf - $m$ ausgeht, nicht auf -mês : so im Ker. gloss., hymn., $\mathrm{Kb}$, R., Sg. 70 (5 mal, nur einmal pirumes gl. I, 766, 27), K. (neben pirumes). Das hängt damit zusammen, dass die sprechenden dieses verbum von ältester zeit her als eine art von präteritopräsens empfanden, weshalb denn auch alle weitere entwickelung darauf hinausläuft, das präteritale aussehen schärfer hervortreten zu lassen. Vgl. nord. erum eruð eru mit várum

1) Im conj. praes. des verb. subst. kommt sîmês nur bei 0. vor, alle übrigen denkmäler haben sin (Graff I, 482). 
váruð váru, got. sium siup mit bêrum bêrup; im nord. heisst es später auch im sg. er ert er wie var vart var. Aus dem ahd. ist hier zu nennen die 3. pl. sintun bei Is. frg. mit der präteritalen endung der 3. pl. Vielleicht hat in der 1. pl. praes. ind. des verbum subst. nie die volle endung $-m a_{2} s i$ gestanden, so dass bereits die indog. grundform ${ }^{*}$ smés gewesen wäre. Darauf beruht aber ganz direct unser pirum, vgl. beitr. VI, 572 .

Anmerkungsweise sind noch einige kleinigkeiten zu erörtern. 1. Es gibt bei 'T. und in einigen andern denkmälern formen, in denen -mês an eine fertige 1. pl. auf $-n$ angetreten ist. Diese machte seiner zeit Kuhn zum beweise für seine theorie geltend, indem er darauf hinwies, dass ja hier -mês klärlich ein junges anhängsel sei. Darin hat er zweifellos recht, aber es sind merkwürdiger weise nur solche tempusformen, in denen von alter zeit her dic secundäre endung ihren platz hatte. Bei ' $\mathrm{T}$. kommen nemlich mit einer einzigen ausnahme nur präteritalformen in betracht (gabunmes, quamunmes, gisahunmes, gihalotunmes), wonach daun die eine präsensform comenmes analogisiert ist, und ausserdem begegnet nur einmal im cod. Vind. 2732 (gl. I, 704, 4) pirunmes (nicht wie Graff angibt in Ib.): wie man sieht, sämmtlich formen, in denen die älteren denkmäler $-m$ hatten. Dieses war bereits $\mathrm{zu}-n$ ge- worden, als vom präsens her -mês eingeschleppt wurde. 2. In einigen jüngeren quellen begegnet -mas für -mês, z. b. in den Augsb. gl. uuidirneozamas gl. I, 383, 59; beispiele aus Freisinger glossen bei Graff II, 580. Dieselben denkmäler haben auch blintar fur blintêr, habat fur habêt, habata für habêta, kurz, die $\hat{e}$ in endsilben sind hier lautgesetzlich in a ubergegangen. 3. Die funf vorkommenden -mus für -mês (zuspaltemus Pa. 112,15; pespurnemus cod. Vindob. 1239 gl. 1, 787, 26 und 3 fälle bei OF.: bittemus I, 28, 1; singemus I, 6, 15; ilemus V, 23, 76) beruhen auf verschreibung. In Pa. ist zaspallumes zu lesen = zispaldumes gl. K., denn die gemeinsame vorlage (das original?) hatte wahrscheinlich durchweg wie die S. Galler hs. -umes (s. u.). $\mathrm{Ob}$ auch die zweite form in pespurnumes zu bessern ist, bleibt fraglich, da -umês im präs. ausser dem Ker. gl. nicht vorkommt. $u$ ist in geheimschrift gegeben und der schreiber kann sich dabei leicht geirrt haben im gedanken an die endung des lat. verbs. Versehen werden auch vorliegen in den drei stellen 
der Freisinger Otfriedhs.; walıscheinlich war dem schreiber die endung -mês nicht mebr gelïufig, er schrieb sie daher nur mechanisch aus der vorlage ab und an einigen stellen kam $\mathrm{ihm}$ die ihm gewohntere lat. endung in die feder. 4. Zahlreicher sind die formen auf -men; ich will die welche mir zur hand sind auffuhren, damit jeder sich selbst ein urteil uber diese verschreibungen - denn weiter sind sie nichts - bilden kann. Die meisten finden sich in dem grossen in 17 handschriften uberlieferten bibelglossar (Clm. 18140. 1944). Vind. 2723. 2732 u. s. w.), und zwar stets an solchen stellen, wo

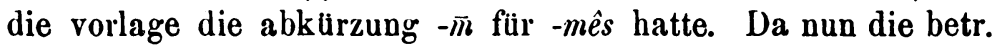
schreiber die endung -mês in ihrer lebendigen sprache nicht mehr kannten, sondern statt dessen nur -en, und da es ihnen geläufig war, den strich durch -en aufzulösen, so verfielen sie auf den leicht begreiflichen fehler, - men für $-\bar{m}$ zu setzen. Die stellen sind:

Gl. I, 478, 18 leitamen (gerimus) Gotw. 103: leitōn Vind. 2723.

Gl. I, 483, 29 ziomen Clm. 18140. 19440: ziohom Vind. 2723. 2732.

Gl. I, 491, 41 uuerdemen Clm. 18140: uuerdē̄ Clm. 19440.

Gl. I, 491, 41 uuerdamen Vind. 2732: uuerdaī Vind. 2723.

Gl. I, 503, 39 pismahetomen Clm. 18140: pismahetom Vind. 2732. Clm. 19440.

Die von Steinmeyer in Haupts zs. 15, 50 beigebrachten belege arsuochemen, lazamen, scidomen aus den sehr jungen Tegernseer Virgilglossen sind ganz sicher ebenso zu beurteilen. Einige andere formen, die Graff II, 580 aus jungen glossen anfuhrt, entziehen sich vorläufig der genauereu beurteilung, ich zweifele aber keinen augenblick, dass es sich damit genau so verhält, wie mit den genannten.

So geht denn unsere rechnung glatt auf. In betracht zu ziehen sind nur $-m \hat{c} s$ und $-m$, die functionell verschieden sind; jenes ist die alte primärform $=$ urspr. $-m a_{2} s i$, dieses die alte secundärform $=$ urspr. $-m a_{1} n$. Letztere wird am ausgange der ahd. periode verallgemeinert, wie dies im ostgermanischen schon viel fruher geschehen war.

B) Der thematische vocal im ind. präs. und im adhortatirus. Was zunächst letzteren anlangt, so entspricht das got. 
furam vigam mit seinem $a=a_{2}$ genau dem skr. váhāma, und es kann nicht zweifelhaft sein, dass wir hier $a$ auch im althd. zu erwarten haben. Denn die von Paul IV, s. $363 \mathrm{ff}$. aufgestellte regel, dass got. $-a m$ in den ubbrigen dialekten sich zu -um verdumpfe, kaun unmöglich aufrecht erhalten werden, da $u$ vor nasalen urgermanisch ist und wie obcn gezeigt ist stets auf $a_{1}$ zurückgeht. Paul hat auch nur einen einzigen scheinbar beweisenden fall, das ist der d. pl. got. dagam im verhältnis zu ahd. tagum; aber hier ist die schwache stammform *dageam platze und aus einer grundform *dáge-me(z) konnte nur die in allen germ. sprachen ausser dem got. wirklich vorliegende form dagum hervorgehen. Im got. ist wie in andern sprachen die schwache stufe durch die starke verdrängt, daher dagam, während nord. dogum ags. dagum ahd. tagum lautgesetzlich aus der grundform entwickelt sind. Diejenigen ahd. denkmäler, welche die form des adhortativus noch nicht durch die 1. pl. opt. ersetzt haben, zeigen nun in der tat -am, meist jedoch schon mit der vom ind. herkommenden prinären suffixform -amês:

$\mathrm{Rb}$. ingagan kisezzames (opponamus) gl. I, 474, 19; camiscames (confundamus) 316, 27; niozzames (abutamur) 388,62

S. Paul. farames (transeamus) gl. I, 732, 11

R. zaprechames (disrumpamus) gl. I, 113, 15

Clm. 18140. pisuihhan (circumveniamus) gl. I, 815, 47; missiniozan 386, 65; inkinnames (aperiamus) 673, 60

Gotw. 103. uzuninnames (avellamus) gl. I, 599, 5

Weiss. Kat. gilaubames endi bijehames (credamus et confiteamur).

Dazu kommen 9 beispiele aus der benedictinerregel, die Seiler beitr. I, 452 zusammengestellt hat, und einige aus 0 ., von Kelle in Haupts zs. 12, 41 gesammelt: farames I, 18, 33. III, $23,55.57$.

Im indicativ sind die erreichbar ältesten ahd. formen die des Keronischen glossars. Hier herscht bekanntlich beim starken verbum sowol als beim schwachen erster classe -umês (uber d. Ker. gl. s. $181 \mathrm{ff}$.), und damit stimmt das nordische uberein (bindum, forum). Urgermanisch haben wir also anzusetzen */äremasi, woraus nach den oben dargestellten laut- 
gesctzen noch im gemeingerm. * farumais $(i)$ sich entwickelt hat, denn $e$ vor $m$ in unbetonter silbe wurde $\mathrm{zu} u$. Ob $a_{1}$ vor dem zweisilbigen suffixe $-m a_{2} s i$ bereits indogermanisch ist, lasse ich hier unerörtert, verweise aber auf lat. vehimus altbulg. nesemŭ (serb. pletemo) lit. neszeme, die $a_{1}$ auch für das germanische sicher stellen.

Das älteste althd. hat also farumês im ind., faram im alhort. gehabt. In der folgezeit glichen sich nun die beiden formen aus, indem der adhort. sein $a$ dem indic. gab, dieser aber -mês auf den adbort. übertrug. Die weiteren schicksale des adhort. wurden daher auch für den indic. bestimmend.

Es ist eine in vielen sprachen zu beobachtende erscheinung, dass der alte adhortativus verdrängt wird durch die von uralter zeit her daneben bestehende concurrenzform, der 1. pl. des conjunctivs (vgl. Delbrück, synt. forsch. I, $109 \mathrm{ff}$.), oder, wo der conjunctiv verloren ist, des optativs. Wir beobachten dies im griechischen, lateinischen und slavischen, wo ja bekanntlich keine eigene form des adhort. mehr vorhanden ist. Diesen sprachen schliesst sich nun das althd. des ausgehenden achten jahrbunderts in allen dialekten an. Das alte faram(ess) wird durch die optativform farêm ersetzt, die nun auch gleich die primäre endung crhält, also farêmês oder vielleicht schon früh faremês, denn das mittlere $e$ wird in der B.-R. nie doppelt geschrieben. Da nun aber vor dieser ubertragung der adhortativ die gleiche form wie der indic. gehabt hatte, so sprach man jetzt auch im indic. farêmês oder faremês; denn die sprachen lieben es nicht, einmal zusanmengefallene formen wider zu differenzieren, da ja eben das gefuhl für ihre verschiedenheit verloren gegangen ist.

Den typus faremes finden wir in den meisten ahd. denkmälern herschend, z. b. in Pa, Ra, Is., Frg., T. und in den meisten jüngeren glossen. Die hymmen haben $11 \mathrm{mal}$ das ältere -amês, 16 mal schon -emês, aber davon $11 \mathrm{mal}$ bei schwachen verben, wo $e$ auch aus $j a$ hervorgegangen sein kann. Rb. kennt -emês noch nicht, sondern nur -amês bei starken und schwachen rerben. Das alte denkmal Sg. 70 hat nur zwei -amês (kistritames gl. I, 766, 38; nifiruuerdames 766, 30). In jiingeren bair. glossen ist mir -amês begegnet in uzlesames gl. I, 808, 27; ginuinnames 441,30 ; uallaim $\delta() 7,15$; gihuccames 395,50 ; ir- 
leitlaim 786, 17; vgl. uzlesan (colligimus) 808,28 . In allen diesen glossensammlungen überwiegt aber schon bedeutend -emès; bei schwachen verben begegnet auch -imes mit secundarer verengung von ie zu $i$, z. b. leccim 785, 46; gileccimes 611,28 ; irleittimes 786, 17. Auch im optativ: pilickimes (cognoscamus) 305,39 ; girrimes (destruamus) 528, 7 ; muntrimes (suscitemus) 599 , 1, wodurch die verkürzung der mittelsilbe direct bewiesen wird, da nur ie, nicht aber iê sảmprasarana erleiden kann.

Der annahme Pauls (Beitr. IV, 365), dass -amês lautlich aus -umês entwickelt sei, steht die tatsache hindernd im wege, dass die älteren denkmäler $u$ in mittelsilben nicht zu $a$ abschwächen, sondern unverändert erhalten; z. b. flectieren die hymnen stets atumes atume atumu, während sie doch -amês haben. Dazu kommt, dass im präteritum -umês, wo es vorkommt, nur selten und sehr spät in -amês ubergegangen ist, vgl. qhuatumes B.-R., mitumes Ja, azumes uzuиurphumes uuizumes forliezumes mugumes gifiengumes fundumes u. s. w. T. (Graff II, 579 f.), sculumes O., obwol in diesen denkmälern -umês im präsens nie mehr begegnet. Endlich fehlt * piramês für pirumês gänzlich (Graff II, 14). Ebensowenig wie diese ansicht Pauls ist das haltbar, was ich selbst Ker. gl. s. 182 über die entstehung von -amês aufgestellt hatte.

Die oben s. 129 aufgezählten kurzen indicativformen werden mit $\hat{e}$ anzusetzen sein: oblazêm furlâzz'm bittêm quedêm, denn sie entsprechen genau den Notkerschen indicativen auf $-\hat{e} n$ wie héizên, deren länge gesichert ist (Braune, beitr. II, 137 f.). Bei den secundärformen konnte naturlich eine verkürung des $e$ nicht eintreten. Ich bemerke ubrigens ausdrücklich, dass für die ältere zeit die verkürzung der mittelsilbe in faremês nicht bewiesen ist, dass aber ebensowenig ein zeugnis fur die länge des $\hat{e}$ in opt. farêmês, wie man gewöhnlich schreibt, beigebracht werden kann. Man muss also entweder beidemal faremês oder beidemal farêtês schreiben, und mit secundärer endung beidemal farêm oder farên. 
2. Die endung der zweiten person pluralis.

Hier liegen dic verhältnisse weit einfacher als bei der ersten person. Dennoch ist bisher manches verkannt worden. Die endung ist im indicativ wie im imperativ, also primär und secundär, ursprïnglich $-t a_{1}$ gewesen, wie hervorgeht aus skr.

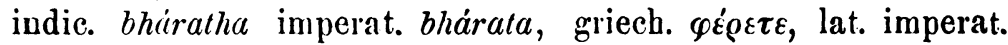
vehite, ir. -berid, altb. vezete, lit. vézate. Es fragt sich nur, was aus *bherete in germanischen lautgesetzlich entstehen musste. Paul in den beitr. IV, 404. VI, $79 \mathrm{f}$. entscheidet sich fur * berep, er meint also, $e$ sei ohne vorber zu $i$ geworden zu sein abgefallen. Das ist mir sehr unwahrscheinlich im hinblick auf die 2. sg. des imperativs. Hier führt ahd. alts. nin mit notwendigkeit auf *nimi aus * neme, denn die annahme einer analogiebildung stösst auf die grössten schwierigkeiten. Weshalb sollte denn * nem verdrängt sein, da es zu der 2. sg. ind. nimis in gar keinem bezuge steht? Die ausgleichung aller personen des imperativs im nord. ags. und fries. (vgl. Gunther, die verba im altostfriesischen Leipzig 1880 s. 31) ist dagegen sehr einfach zu begreifen; denn diese sprachen streben im verbum überhaupt nach möglichster gleichförmigkeit, besonders das nord., welehes ja auch im ind. 2. sg. gefr fur älteres * gifr hat eintreten lassen. Uebrigens ist beim germ. verbum die annahme einer ausgleichung alter verschiedenheiten von vornherein weit wahrscheinlicher, als die annahme der herbeifuhrung einer neuen differenz. Auslautendes $e$ wurde also im urgermanischen ehe es abfiel zu $i$ und wirkte wie urspringliches $i$ auf die stammsilbe. Ob dieses lautgesetz nur fur rein auslautendes $e$ gilt, oder auch $\mathrm{ftr}$ gedecktes, worauf manches namentlich in der behandlung der alten $s$-stämme hinweist, lasse ich hier ununtersucht.

Aus altem *bircte ging also schon im urgermanischen *birip(i), aus *némete nimip(i) hervor. Diese formen sind im got. bairip, nimip erhalten, die endung -ip auch im nord. gefiठ fario, der stammvocal ist aber hier den andern beiden pluralpersonen angeglichen.

Im althochd. haben wir noch ein einziges sehr altes denkmal, welches die alten formen in genügender anzahl aufweist, das sind die Monseer bruchstücke. Im glossar zu der ausgabe 
hat Massmann dicse wichtigen reste \%usammengestellt (S. 37), leider sehr unvollständig und fehlerhaft. Von neuem hat sie dann Paul beitr. IV, 403 gesammelt (im ganzen zwölf belege). Am wichtigsten sind diejenigen belege, wo das $i$ der endung auf die stammsilbe gewirkt hat: ir quidit (dicitis) 16, 8; gasihit (videbitis) 6, 27; ferit (ite) 13, 20; in forstantit (intelligetis) 6, 27 ist der umlaut noch nicht eingetreten. Diese formen sind von dem verdachte einer analogiebildung vollkommen frei und andere erklärungen als die oben gegebcne sind rundweg ausgeschlossen; denn was Paul a. a. o. vorbringt, um diese formen aus der welt zu schaffen, steht auf schwachen füssen. Eine verwechselung mit der dritten singularis liesse sich viclleicht annehmen, wenn es sich um eine einzelne stelle in einem schlecht ubersetzten und nachlässig abgeschriebenen denkmale der ältesten zeit handelte, nicht aber an zahlreichen stellen dieser guten abschrift vorzüglicher iibersetzungen. Die 2. pl. auf -et wie quedet gasehet faret waren gewiss dem schreiber nicht geläufig, aber er verstand sie doch gewiss in zusammenhange der stelle ganz gut. Dazu kommt, dass diese -it auch in der 2. pl. des imperativs stehen, wo ja Pauls erklärungsversuch gar nicht anwendbar ist. Somit liegen hier die ältesten lautgesetzlich den urgermanischen entsprechenden formen der 2 pl. vor. Der indic. präs. eines verbs wie quedhan wurde also in ältesten althochdeutsch so flectiert: quidhu, quidhis, quidhit, quedhumês, quidhit, quedhant; imperat. quidh, quedham, quidhit.

Der grund, weshalb die 2. pl. quidhit verdrängt wurde, liegt im stammsilbenvocalismus; denn wie im singular, so verlangte man auch im plural in bezug hierauf gleichheit der drei personen. Ehe wir uns zur näheren darlegung dieser formubertragung wenden, wird es gut sein, die denkmäler auf die 2. pl. ind. und imper. hin zu durchmustern.

Im Ker. gloss. fehlen indicativformen. Der imperativ hat, soweit er nicht gleich der 3. pl. ind. ist, in Pa. und gl. K. -et, in Ra. auch zweimal -at (vgl. üb. d. Ker. gl. s. 185).

Die Ben.-R. hat ebenfalls nur imperative und diese gehen auf -at aus (Seiler, beitr. I, 452).

In $R$ b. begegnen im ind. zwei -et (ir bauuiset vitabitis 
gl. I, 353, 22; pluazzet libabitis 336,66 ), sonst bei starken und schwachen verben (I. cl.) nur -at, nemlich im ind. hinchat 447, 61 ; faruuera/fat 409, 21; uuesat 363, 47; denchat 380, 15; fuarrat 621,24 ; kiharindat 410,34 , und im imperativ farnemat 412,15 ; pilecat 552,20 ; spriuzat 552, 16 ; kichunicchat 620,24 ; farzimbarat 354, 3.

In $\mathrm{Rd}$. Ib. finden sich in ind. 2 -et, 3 -al, nur bei starken verben: uz pringet 278, 26; artuuelet 284, 54; meizzat snidat 282,19 ; midat 295, 1 . Im imper. kommen nur schwache verba vor und diese liefern nur einen beleg auf -at (uabat Rd. 278, 4), die iibrigen auf -et: piuuerrel 286,67 ; arfuarret 271,51 ; dicket Ib. 280, 71 (= kidiket Rd.); uabet Ib. 278, 4.

Ia bietet inchinnet (nitimini, indic.) 511, 39; furihtel (reveremini, imperat.) 587, 42.

S. Paul. hat findat ir (invenietis) 731, 46.

Aus andren al. glossen fubre ich an firmeldet (imper.) 697, 3 (Sg. 299); dicchet intercedite (frg. S. Paul. 312, 73; firmeldat (imper.) A. 697, 3; unerdat (indic.) 724, 10.

In den bairischen denkmälern ist -at selten. Ich finde nur singat (jubilate) R. 195, 22; girrat (sollicitatis) 326, 48 (Gotw. 103); aritalat (imper.) 523, 19 (Clm. 18140. 19440); zuouahat (arripite) 398, 36 (Clm. 14689). Das herschende ist im indicativ und imperativ -et, z. b. artuelel 379, 30 (Clm. 18140); snidet 368, 32 (Vind. 2723. 2732); irratet 386, 3; kapintet (colligate) R. 61, 32; uueset R. 131, 25; dennet (extendite) R. 131, 33; arhefphet (extollite) R. 131,32. Die sehr zahlreicben belege aus dem grossen bibelglossar (Clm. 18140 u. a.) bier alle aufzuzählen lohnte sich nicht. In jüngeren denkmälern begegnet Ubrigens wider -it, z. b. irratit 386, 3 (Clm. 13002); hinchit 440, 23 (Gotw. 103. Clm. 13002); uuanchit (claudicatis) ebenda (Clm. 14689); nerit 527, 17 (Clm. 13002); pigoumit (videte) 795, 8 (Clm. 18140); chundit (praedicate) 442, 1 (Clm. 13002); firnemit 606, 5. 742, 27. Auch im optativ: uuerfit (ut .. ordiremini) 607,28 (Clm. 14689); ubertrinchit (ut . a aestuetis) 597, 66 (Clm. 13002. 17403) = upirtrinchit Clm. 14689; cherit (non deflectatis) 564, 53 (Clm. 13002. 17403) = kerit Clm. 22201; dultit (agatis) 698, 26 (Clm. 22201). 
Den fränkischen denkmälern fehlt -at so gut wic vollständig. Es herscht hier wie im bairischen -et. So bei Is. und $\mathrm{O}$. ausnahmslos; wenn $\mathrm{T}$. einige wenige -at aufwcist, so kommen diese sicher auf rechnung eines alemannischen schreibens (Paul beitr. IV, 404). In den glossen begegnet einmal -it (aruuntid adnuntiate cod. Wirzeb. 623, 8), sonst -et: nemet (imper.) 795, 15 (cod. Berol.); helphet (subvenite) cod. Wirzeb. 621,27 ; irhúcget (retinetis) 775, 11 (Wo. 3).

Aus dieser ubersicht iiber das belegmaterial geht hervor, dass -at specifisch alemannisch ist (denn die wenigen - at in bair. quellen kommen wol zweifellos auf rechnung al. vorlage), während auf bairischem und fränkischem gebiete -et berscht. Die -et in alemannischen quellen (wozu ich die handschriften des Ker. glossars nicht rechne) machen den eindruck einer erhaltenen altertlimlichkeit; vielleicht hat daher auch hier einmal -et bestanden. Freilich sind dabei nur die starken verba in rechnung zu ziehen, denn bei den schwachen 1. cl. kann $e$ auf ja zuriickgehen.

Keine schwierigkeit bietet der erklärung die alem. form auf -at: diese kann ibr a nur aus der 1. pl. auf -amès, dem alten adhortativus, haben, nachdem der stammvocal bereits den andern beiden pluralpersonen angeglichen war. In allen denkmälern, in denen -at vorkommt oder herscht, begegnen denn auch in entsprechender anzahl die 1. pl. auf-amês. Schwieriger ist die weit verbreitete form auf -et zu erklären, die, wie wir oben gesehen haben, keineswegs die lautgesetzliche fortsetzung der 2. pl. imper. (griech. $\lambda \varepsilon^{\prime} \gamma \varepsilon \tau \varepsilon$ ) ist. Die entscheidung wurde leichter sein, wenn wir wllssten, ob die endung kurzen oder langen vocal hat. Ich meinesteils sehe keinerlei möglichkeit, diese frage $z u$ entscheiden, da uns die B.-R. und Notker (wo -et aus -at entstanden ist) im stiche lassen. Ist -êt zu schreiben, so haben wir darin die optativform zu erblicken, die tiber den imperativ in den indicativ gedrungen ist; diese formulbertragung war ja sehr leicht, da die ersten personen wenigstens in einigen dialekten schon gleich gemacht waren. Oder ging die zweite person voran? Das ist ganz wol möglich, denn -et ist weiter verbreitet als -emês. Ist aber -et kurz, und das ist doch die einfachere annahme, so haben wir in beret die alte zweite person des duals zu erblicken, urgerm. * béredam $=$ ind. 


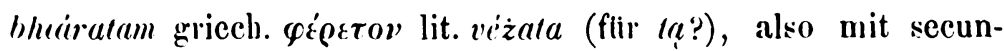
däurer suffixform, die sich dann rom imperativ aus auf den inclicativ verbreitet haben muss. Das eintreten einer dualform fuir die entsprechende pluralform hat sein analogon im lateinisschen, wo vehitis, wie Baunack in Curtius stud. bd. $\mathrm{X}, 61$ gezeiigt hat, $=$ skr. váhathas, also ein alter dual ist. Dann hätte alsio im althd. von ältester zeit her nemet und nimit nebeneinander bestanden. Diese erklärung der endung -et scheint milr der wahrheit am nächsten zu kommen. LEIPZIG, 27. nov. 1881 . RUUOLF KÖGEL. 